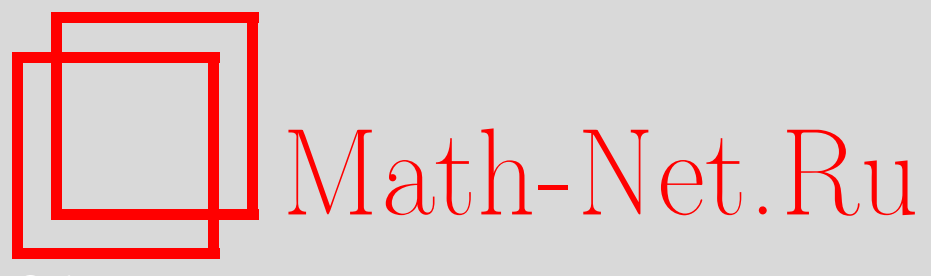

В. М. Челноков, В. Л. Зефирова, Матричный показатель степени принадлежности узла сети сердцевине сети, $\mathrm{Ma}$ тем. заметки, 2006, том 80, выпуск 6, 908-919

DOI: https://doi.org/10.4213/mzm3366

Использование Общероссийского математического портала Math-Net.Ru подразумевает, что вы прочитали и согласны с пользовательским соглашением http://www . mathnet.ru/rus/agreement

Параметры загрузки:

IP : 54.147 .182 .235

26 апреля 2023 г., 14:17:00

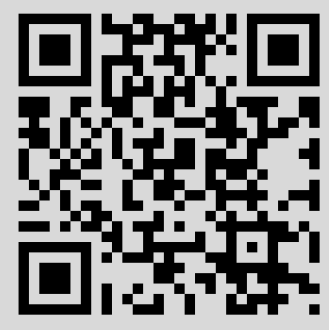




\section{МАТРИЧНЫЙ ПОКАЗАТЕЛЬ СТЕПЕНИ ПРИНАДЛЕЖНОСТИ УЗЛА СЕТИ СЕРДЦЕВИНЕ СЕТИ}

\section{В. М. Челноков, В. Л. Зефирова}

В центре внимания статьи находятся координаты собственных векторов матрицы связей сети, правого и левого, соответствующих максимальному собственному значению матрицы. Показывается, что каждая координата измеряет маршрутную центральность положения соответствующего узла в связочной структуре сети и статус его узлов-соседей, в силу чего она выражает степень принадлежности узла к сердцевине этой структуры - его структурную сердцевинность. И соотнесение координат векторов с положением узлов, и само вычисление координат опирается на итерационный степенной метод. В статье показывается обоснованность его применения для сетей различной структуры. Рассматриваются возможные приложения и числовой пример с реальной сетью большого размера (197 узлов, 780 связей).

Библиография: 17 названий.

\section{Введение}

В статье показывается, что правый и левый собственные векторы сетевой матрицы, которые соответствуют ее максимальному собственному значению, измеряют степень принадлежности узлов сети к сердцевине сети, т.е. служат, в наших терминах, измерителями структурной сердиевинности узлов. Эта сердцевинность, представленная координатами векторов, является функцией на множестве узлов сети. Узлы упорядочиваются по убыванию значений этой функции; тем самым, как мы показываем, они выстраиваются в порядке ослабления структурных параметров, указывающих на принадлежность узла сети к сердцевине сети. Некоторая группа узлов из верхней части рейтинга принимается в качестве сердцевины сети; кроме того, любые два узла сети можно сравнить по этой шкале друг с другом, и это не менее важно, чем выявление сердцевины.

В каждой ориентированной сети присутствует сердцевинность двух типов: $i$-я координата правого вектора показывает out-сердцевинность положения узла $i$ в связочной структуре сети, а $i$-я координата левого вектора дает іп-сердцевинность его положения в этой структуре. Первая величина отражает суммарный вес маршрутов, начинающихся в данном узле, вторая - вес маршрутов, заканчивающихся в нем. Взаимосвязь маршрутов и собственных векторов объясняется тем, что, с одной стороны, $(i, j)$-элемент $k$-й степени сетевой матрицы равен весу (числу) маршрутов 
сети от узла $i$ к узлу $j$ длиною $k$ связей, а, с другой, эти степени матрицы фигурируют в итерациях при нахождении собственных векторов так называемым степенным методом. В неориентированной сети оба типа сердцевинности сливаются в один.

Рейтинги узлов сети по out / in-сердцевинности под разными названиями применяются в социальных сетях, сетях цитирования, гипертекстовых и других сетях. Рейтинг по in-сердцевинности, в случае стохастической сетевой матрицы, совпадает с финальным распределением цепи Маркова - случайного блуждания по сети. Мы приводим примеры использования показателя в моделях понимания текста и в опытах по формированию неклассических категорий.

Мы даем обоснование вычислительной схемы, приводящей к векторам сердцевинности. Работы по матричному анализу, на которые мы опираемся, не содержат нужных нам сетевых интерпретаций. С другой стороны, в тех разрозненных публикациях, где описываются применения показателей, вычисляемых так же, как наши, обычно отсутствуют, во-первых, основные сведения касательно математической стороны дела и, во-вторых, упоминания об общесетевом характере понятия сердцевинности. Своей статьей мы хотим помочь устранить этот разрыв.

Теоретические представления у нас реализованы в виде компьютерных программ. В статье приводится два числовых примера, один из которых использует реальную сеть большого размера (197 узлов, 780 связей).

\section{1. Основные обозначения}

Рассмотрим сеть на узлах $1,2, \ldots, n$ со взвешенными и направленными, в общем случае, связями. Сеть представляется матрицей связей $A$ размера $n \times n$, в которой элемент $A(i, j)>0$ равен весу связи $(i, j)$, если таковая присутствует, и равен нулю, если она отсутствует. Когда веса всех связей одинаковы, имеем стандартную матрицу смежности сети (графа), состоящую из нулей и единиц. Таким образом, $A$ - вещественная, неотрицательная, квадратная и, в общем случае, несимметричная матрица.

Как хорошо известно, для всякой вещественной матрицы $A$ наборы собственных значений (спектры) $A$ и транспонированной $A^{\prime}$ одинаковы, и, если $\bar{x}$ и $\bar{y}-$ собственные векторы-столбцы матриц $A$ и $A^{\prime}$ при одном и том же собственном значении $\lambda$, т.е. $A \cdot \bar{x}=\lambda \cdot \bar{x}$ и $A^{\prime} \cdot \bar{y}=\lambda \cdot \bar{y}$, то $\bar{x}$ и $\bar{y}$ называют соответственно правым и левым собственными векторами матрицы $A$, отвечающими собственному значению $\lambda$, имея в виду, что $\bar{y}^{\prime} \cdot A=\lambda \cdot \bar{y}^{\prime}$, где $\bar{y}^{\prime}$ - вектор-строка [1], [2]. Собственные значения матриц $A$ и $A^{\prime}$ нумеруются в порядке невозрастания модуля: $\left|\lambda_{1}\right| \geqslant\left|\lambda_{2}\right| \geqslant\left|\lambda_{3}\right| \geqslant \cdots \geqslant\left|\lambda_{n}\right|$ (каждое число $\lambda$ присутствует в этом перечне столько раз, какова алгебраическая кратность $\lambda$ ); собственные векторы этих матриц, отвечающие $\lambda_{s}$, обозначаются $\bar{x}_{s}$ и $\bar{y}_{s}, s=1,2,3, \ldots, n$; число $r(A)=\left|\lambda_{1}\right|$ называют спектралъным радиусом $A$ (и $A^{\prime}$ ).

Ввиду частого употребления терминов "собственный вектор" и "собственное значение", мы будем использовать сокращения с.в. и с.з.

\section{2. Степенной метод}

Для произвольной вещественной матрицы число $\lambda_{1}$ и векторы $\bar{x}_{1}, \bar{y}_{1}$ могут быть найдены при помощи степенного метода, одного из наиболее известных в матричных вычислениях [1]-[4]. В нашем, “сетевом" случае данный метод не только служит 
указанной цели, но также позволяет увидеть связь координат $x_{1}(i), y_{1}(i)$ с положением (сердцевинностью) узла $i$ в связочной структуре сети.

Предположим, что максимальное по модулю с.з. матрицы $A$ имеет алгебраическую кратность 1 и единственно, т.е. $\left|\lambda_{1}\right|>\left|\lambda_{2}\right|$; предположим также, что оно вещественно. Пусть $\bar{z}_{0}-$ вектор размерности $n$, не ортогональный $\bar{x}_{1}$, и $\nu_{k}=\left\|A^{k} \bar{z}_{0}\right\|$, где $\|\cdot\|$ - некоторая векторная норма, например, евклидова длина. Метод базируется на том, что при данных предположениях вектор $\bar{z}_{k}$ в итерациях

$$
\bar{z}_{k}=\nu_{k}^{-1} A^{k} \bar{z}_{0}, \quad k=0,1,2, \ldots,
$$

стремится к вектору $\left\|\bar{x}_{1}\right\|^{-1} \bar{x}_{1}$ при $k \rightarrow \infty$, а отношение $\nu_{k} / \nu_{k-1} \rightarrow \lambda_{1}$. В более удобной для вычислений записи,

$$
\bar{z}_{k+1}=\alpha_{k}^{-1} A \bar{z}_{k}, \quad k=0,1,2, \ldots,
$$

где $\alpha_{k}=\left\|A \bar{z}_{k}\right\| \rightarrow \lambda_{1}, \alpha_{0} \alpha_{1} \cdots \alpha_{k-1}=\nu_{k}$.

На практике, можно задать малое число $\varepsilon, 0<\varepsilon<1$, и, вычисляя на каждом шаге

$$
e=1-\frac{1}{n} \sum_{i=1}^{n} \frac{z_{k}(i)}{z_{k-1}(i)},
$$

продолжать итерации, пока $|e|>\varepsilon$. Итераций потребуется тем меньше, чем больше $\left|\lambda_{1}\right| /\left|\lambda_{2}\right|$ (см. [2]).

Аналогичные вычисления с матрицей $A^{\prime}$ дают вектор $\bar{y}_{1}$.

Сходимость метода обеспечивают сделанные предположения касательно $\lambda_{1}$. Их обоснованность будет рассмотрена ниже. Заранее скажем, что в нашем случае всегда $\lambda_{1}>0$, т.е. спектральный радиус $r=\lambda_{1}$, и все координаты векторов $\bar{x}_{1}, \bar{y}_{1}$ положительны $\left(\bar{x}_{1}>0, \bar{y}_{1}>0\right)$. Отсюда, в частности, следует, что в качестве $\bar{z}_{0}$ можно взять любой неотрицательный ненулевой вектор.

Заметим, что подобные вычисления с матрицей, редуцированной определенным образом, позволяют находить остальные с.в. и с.з. (метод исчерпывания или дефляции [3]).

\section{3. Показатель сердцевинности}

Если два узла сети занимают одинаковое структурное положение, т.е. симметричны друг другу, то в векторе $\bar{x}_{1}$, как и в векторе $\bar{y}_{1}$ (а также в любом другом с.в. сетевой матрицы) координаты, отвечающие этим узлам, одинаковы. Если же какие-то две координаты, скажем вектора $\bar{x}_{1}$, различны, то соответствующие два узла имеют разное положение в структуре сети. Формулы (1) и (2) позволяют видеть две структурные особенности узла, которые проявляются тем сильнее, чем больше его координата в $\bar{x}_{1}$ или $\bar{y}_{1}$. Исходя из них, координату можно трактовать как степень принадлежности узла к сердцевине сети - как сердцевинность узла.

3.1. Сердцевинность как центральность. Обозначим $(i, q)$-элемент матрицы $A^{k}$ через $A^{k}(i, q), k=1,2, \ldots$. Этот элемент есть, как известно, суммарный вес различных маршрутов длиною $k$ связей, каждый из которых начинается в узле $i$, а заканчивается в узле $q, i, q=1,2, \ldots, n$; когда вес каждой связи в сети равен 1 , это просто количество $(i, q)$-маршрутов. Данный факт хорошо известен в теории 
графов; он вытекает непосредственно из определения матричного умножения. Напомним, что марируm из $i$ в $q$ - это последовательность связей сети такая, что началом первой связи является узел $i$, началом каждой следующей связи служит узел-конец предыдущей связи, а концом последней связи является узел $q$; при этом любой узел и любую связь маршрут может проходить любое число раз. Вес маршpyma длиною $k$ связей определяется как произведение весов этих $k$ связей.

Обозначим, далее,

$$
S_{\text {out }}^{(k)}(i)=\sum_{q=1}^{n} A^{(k)}(i, q), \quad S_{\text {in }}^{(k)}(i)=\sum_{q=1}^{n} A^{(k)}(q, i)
$$

- вес (количество) маршрутов длины $k$, имеющих узел $i$ начальным и конечным пунктом соответственно; в частности, $S_{\text {out }}^{(1)}(i)=d_{\text {out }}(i), S_{\text {in }}^{(1)}(i)=d_{\text {in }}(i)-$ cmenень исхода и степень захода узла $i$.

Теорема 1. Пусть сетевая матрица А имеет с.з. $\lambda_{1}=r>\left|\lambda_{2}\right|$ u c.в. $\bar{x}_{1}>0$, $\bar{y}_{1}>0$. Тогда для всякой пары $i, j$ узлов сети, при любом достаточно большом $k$ справедливо:

(a) если $x_{1}(i)>x_{1}(j)$, mo $S_{\text {out }}^{(k)}(i)>S_{\text {out }}^{(k)}(j)$;

(б) если $y_{1}(i)>y_{1}(j)$, mo $S_{\text {in }}^{(k)}(i)>S_{\text {in }}^{(k)}(j)$.

ДоказАТЕЛЬСтво. Рассмотрим итерации по формуле (1) с вектором $\bar{z}_{0}$, состоящим из одних единиц. Имеем

$$
\nu_{k} z_{k}(i)=S_{\text {out }}^{(k)}(i), \quad \lim _{k \rightarrow \infty} z_{k}(i)=\frac{x_{1}(i)}{\left\|\bar{x}_{1}\right\|}, \quad i=1,2, \ldots, n .
$$

Поэтому

$$
\lim _{k \rightarrow \infty} \frac{S_{\text {out }}^{(k)}(i)}{S_{\text {out }}^{(k)}(j)}=\lim _{k \rightarrow \infty} \frac{z_{k}(i)}{z_{k}(j)}=\frac{x_{1}(i)}{x_{1}(j)},
$$

откуда следует справедливость (а). При итерациях же с матрицей $A^{\prime}$ имеем

$$
\lim _{k \rightarrow \infty} \frac{S_{\text {in }}^{(k)}(i)}{S_{\text {in }}^{(k)}(j)}=\frac{y_{1}(i)}{y_{1}(j)},
$$

откуда следует справедливость (б). Теорема доказана.

Таким образом, чем больше координата $x_{1}(i)$, тем выше ранг узла $i$ как исходного пункта маршрутов сети, и чем больше $y_{1}(i)$, тем выше его ранг в качестве конечного пункта маршрутов. Величины $d_{\text {out }}(i), d_{\text {in }}(i)$ устанавливают эти ранги в начальном приближении.

Следовательно, первая из упомянутых особенностей состоит в том, что $x_{1}$ - и $y_{1}$ координата узла выступают как измерители центральности, важности, нагруженности данного узла. Это те качества, с которыми ассоциируется обычно сердцевина всякой структуры, материальной или мысленной.

3.2. Сердцевина как плотная зона. Предположим, что доля узлов сети, у которых координата $x_{1}$ больше заданной величины, быстро убывает с ростом последней, например, как степенная функция (похоже, что это типично для реальных 
сетей большого размера). Тогда в силу определяющего соотношения

$$
\lambda_{1} x_{1}(i)=\sum_{q=1}^{n} A(i, q) x_{1}(q), \quad i=1,2, \ldots, n,
$$

узлы $i$ с высоким рангом по $x_{1}$ смежны с узлами $q$, также имеющими высокий $x_{1}$ ранг $(A(i, q)>0)$; в меру возможностей сети, они образуют плотную зону - подграф, близкий к полному. Аналогичная ситуация - с узлами высокого ранга по $y_{1}$.

Следовательно, группа узлов высокого ранга по $x_{1}$ или $y_{1}$ выделяется в сети насколько возможно повышенной плотностью, а это характерная черта сердцевины в структурах самой разной природы.

В силу этой и предыдущей особенностей величины $x_{1}(i)$, мы называем eе outсердцевинностью узла $i$, обозначая $x_{1}(i)=c_{\text {out }}(i)$. Величина $y_{1}(i)=c_{\text {in }}(i)$ называется in-сердцевинностъю. В случае неориентированной сети матрица симметрична $\left(A^{\prime}=A\right)$, правый и левый с.в. совпадают и сердцевинность обозначается просто $c(i)$.

Заметим, что известные показатели центральности такие, как обычная степень узла и среднее или максимальное расстояние узла от остальных узлов сети, отличаются от показателя сердцевинности своим "безразличием" к второй особенности: узлам с большой степенью или малым расстоянием не присуща тенденция к взаимной смежности.

3.3. Некоторые применения. Показатель сердцевинности часто фигурировал под другими названиями. Его исторически первое применение относится, видимо, к сетям типа социограмм и турниров ("задача о лидере") [5], [6]. Величина $c_{\text {out }}(i)$ называется там “действительной силой" узла (игрока) $i$. Показатель $c$ под именем сердцевинности (coreness) известен в социальных сетях [7].

Интересен в прикладном отношении случай стохастической сетевой матрицы, когда

$$
\sum_{j=1}^{n} A(i, j)=1, \quad i=1,2, \ldots, n .
$$

Матрица задает здесь конечную цепъ Маркова с переходными вероятностями $A(i, j)$; например, случайное блуждание частицы, которая в каждый дискретный момент $k$ находится в одном из узлов сети, а "между" моментами $k$ и $k+1$ мгновенно переходит по связи в какой-то соседний узел $(k=0,1,2, \ldots)$. Рассмотрим итерации $(2)$ с матрицей $A^{\prime}$ и неотрицательным $\bar{z}_{0}$ таким, что

$$
\sum_{i=1}^{n} z_{0}(i)=1
$$

(начальное распределение). При норме $\alpha$, равной сумме координат вектора, с учетом стохастичности $A$ и данного свойства $\bar{z}_{0}$ имеем $\alpha_{k}=1, k=0,1,2, \ldots$. Поэтому, согласно (2)

$$
z_{k+1}(i)=\sum_{j=1}^{n} A^{\prime}(i, j) z_{k}(j)=\sum_{j=1}^{n} z_{k}(j) A(j, i),
$$

т.е. $z_{k+1}(i)$ равно полной (безусловной) вероятности того, что частица находится в момент $k+1$ в узле $i$. При условиях сходимости (1) и (2)

$$
\lim _{k \rightarrow \infty} \bar{z}_{k}=\bar{c}_{\text {in }}>0,
$$


т.е. вектор $\bar{c}_{\text {in }}$ дает финальное распределение цепи Маркова, причем не зависящее от $\bar{z}_{0}$. Таким образом, узлы in-сердцевины - самое вероятное местонахождение блуждающей частицы в установившемся режиме (величина $c_{\text {out }}$ здесь у всех узлов одинакова).

Эта модель случайного блуждания показывает себя эффективной применительно к поведению пользователя, путешествующего по связям-ссылкам гипертекстовой сети. Она заложена в популярной поисковой системе Google, в ее методе получения рейтинга страниц Всемирной Паутины [8], [9]. Узлы (веб-страницы) с высокой $c_{\text {in }}-$ это "авторитеты", в которые сеть чаще всего приводит путешественников [8].

Рейтинг узлов по $c$ применяют в когнитивной психологии. Согласно модели понимания читаемый текст представляется в памяти человека как сеть из простых предложений (смысловых единиц текста) и ненаправленных смысловых связей между ними. Узлы этой сети с высокими с являются макроединицами данного текста, т.е. несут в нем наибольшую смысловую нагрузку, дают его конспект-реферат. Это подтверждают эксперименты, согласно которым быстрота припоминания текстовых единиц растет с ростом их $c[10]$.

Другой пример - из когнитивной лингвистики. Изучаемые там неклассические категории (объединения сходных предметов) отличаются от классических сердцевинно-периферийной структурой - градацией своих элементов по степени принадлежности, представительности, “прототипичности”. Применяют следующее "взвешивание" членов категории. Каждому предмету ставят в соответствие некоторую часть атрибутов из общего списка, образуя 2-дольный граф "предметы-атрибуты". Каждый атрибут получает вес, равный числу предметов, которым он приписан. Затем предмет получает вес, равный сумме весов приписанных ему атрибутов [11]. Видно, что данная процедура представляет собой два первых цикла итераций вычисления сердцевинности. Усовершенствование состояло бы в замене данных весов значениями $c$.

\section{4. Обоснование вычислительной схемы}

Истолкование векторов $\bar{x}_{1}, \bar{y}_{1}$ как показателей сердцевинности опирается на предположения о сходимости итераций (1) и (2), т.е. на указанные свойства числа $\lambda_{1}$, на предполагаемую положительность векторов $\bar{x}_{1}, \bar{y}_{1}$ и на особенности последовательных приближений этих векторов, связанные с видом формул (1) и (2). Обоснованность этих предположений вытекает из классических результатов Перрона и Фробениуса для неотрицательных матриц [1], [6], [12].

4.1. Теорема Перрона-Фробениуса. Как установил Перрон, если вещественная матрица $A$ положительна, т.е. все ее элементы больше нуля, то

(а) ее спектральный радиус $r>0$;

(б) $r$ является с.з. $A$, т.е. $r=\lambda_{1}$;

(в) этому с.з. отвечает положительный с.в.;

(г) с.з. $r$ имеет алгебраическую кратность 1 ; отсюда, с.в. единственен с точностью до скалярного множителя;

(д) $|\lambda|<r$ для всякого с.з. $\lambda$ такого, что $\lambda \neq r$.

Вместе (а), (б), (г) и (д) означают, что $r=\lambda_{1}>\left|\lambda_{2}\right|$. 
Данная теорема обобщается на случай неотрицательных неразложимых матриц таких, чья сеть сильно связна (или просто связна, в случае ненаправленных связей). Для такой матрицы, как доказал Фробениус, справедливы пункты (а)-(г) теоремы Перрона, однако выполнение (д) не гарантируется [1; теорема 8.4.4].

Заметим, что если матрица $A$ неразложима, то и транспонированная $A^{\prime}$ неразложима, поскольку сеть $A^{\prime}$ отличается от сети $A$ лишь противоположным направлением связей.

С.з. $r$ неразложимой матрицы $A$ называют перроновым корнем [1] или доминирующим с.з. [2] матрицы $A$ (и $\left.A^{\prime}\right)$.

Сетевая матрица у нас всего лишь неотрицательна, и нам весьма важно сохранение пункта (д): если существует с.з. $\lambda \neq r$ такое, что $|\lambda|=r$, то сходимость итераций (1) и (2) отсутствует.

Неотрицательные неразложимые матрицы, обладающие только одним с.з., равным по модулю $r$, были выделены Фробениусом под именем примитивных матриц [1], [12].

4.2. Случай неориентированной связной сети. В этом случае сетевая матрица симметрична и неразложима. Всякая сеть является либо однодольной, либо двудольной; в последней, по определению, все множество узлов разбивается на два класса таких, что начало каждой связи лежит в каком-то одном классе, а конец в другом ("многодольность" сети оставляет ее 2-дольной). 2-дольные сети часто используются в приложениях для представления данных.

ТЕОРема 2. Матрица неориентированной связной сети не примитивна тогда и только тогда, когда сеть 2-дольна.

ДокАзАтельство. В самом деле, сетевая матрица $A$ примитивна тогда и только тогда, когда $A^{k}>0$ при всяком, достаточно большом $k$ [1; теорема 8.5.2]. Предположим, что сеть 2 -дольна. Пусть $1,2, \ldots, n p$ - узлы первой доли (" $p$-узлы"), а $n p+1, n p+2, \ldots, n p+n t=n-$ узлы второй доли (" $t$-узлы"). Тогда структура матрицы $A$ такова, что матрица $A^{2 k+1}, k=0,1,2, \ldots$, имеет ненулевые элементы лишь в угловых подматрицах справа вверху и слева внизу, размера $n p \times n t$ и $n t \times n p$ соответственно, в то время как $A^{2 k}, k=1,2, \ldots$, имеет их лишь в угловых квадратных подматрицах слева вверху и справа внизу порядка соответственно $n p$ и $n t$. Следовательно, все степени $A$ содержат нулевые подматрицы, поэтому $A$ не примитивна.

Предположим, что $A$ не примитивна. Пусть $M$ обозначает матрицу смежности графа сети, который отличается от заданной сети лишь тем, что в нем вес каждой связи равен 1. Очевидно, маршруты в сети и графе одни и те же, поэтому при любом данном $k$ неравенство $A^{k}>0$ выполнено тогда и только тогда, когда $M^{k}>0$, т.е. либо матрицы $A$ и $M$ обе примитивны, либо они обе не примитивны. Значит, $M$ не примитивна, т.е., имея с.з. $r(M)$, она имеет еще с.з., не равное $r(M)$, но равное ему по модулю. Таким с.з. может быть только $-r(M)$, так как $M$ симметрична и потому все ее с.з. вещественны. Наличие же такой пары с.з. у связного графа равносильно его 2-дольности [6; теорема 3.4]. Очевидно, “дольность" графа сети и самой сети одинакова. Поэтому сеть 2-дольна.

Теорема доказана. 
Заметим, что несимметричная неразложимая матрица не обязательно примитивна, даже когда ее сеть не 2-дольна. Например, у матрицы смежности ориентированного цикла на трех узлах все три с.з. имеют модуль, равный 1 (два из них комплексные сопряженные числа).

Отметим еще, что в матрицах $A^{2 k}, k=1,2, \ldots$, угловые квадратные подматрицы равны $P^{k}$ и $T^{k}$, где $P$ и $T$ - примитивные матрицы. Действительно, $P$ задает сеть на $p$-узлах, которая связна ввиду связности всей 2-дольной сети и однодольна, так как с.з. ее графа неотрицательны (это квадраты с.з. графа всей 2-дольной сети), из чего следует примитивность $P$. Та же ситуация в случае матрицы $T$ с еe $t$-сетью. С.з. $P$ и $T$ равны квадратам с.з. $A$; координаты их с.в. образуют 2 -дольное разбиение координат с.в. $A$.

4.3. Добавление петель. В практических вычислениях полезно использовать тот факт, что любая неотрицательная, неразложимая матрица, в которой все элементы на главной диагонали положительны, является примитивной [1; лемма 8.5.5]. Поэтому, когда сеть сильно связна (просто связна, при ненаправленных связях), вычисления (2) лучше проводить, например, с матрицей $A+I$, где $I$ - квадратная матрица порядка $n$, в которой $I(i, i)=1$ для $i=1,2, \ldots, n, I(i, j)=0$, когда $i \neq j$. При этом каждое с.з. возрастает на 1 , а все с.в. остаются прежними. Формула (2) тогда приобретает вид

$$
z_{k+1}(i)=\alpha_{k}^{-1}\left[z_{k}(i)+\sum_{q=1}^{n} A(i, q) z_{k}(q)\right], \quad \alpha_{k}=\left\|\bar{z}_{k}+A \bar{z}_{k}\right\| \rightarrow r+1 .
$$

Переход от $A$ к $A+I$ означает снабжение каждого узла сети петлей (связью с самим собой); в результате, сеть утрачивает 2-дольность, если таковая была.

\section{5. Два числовых примера}

ПримеР 1. Мы провели вычисления на компьютере для сети взаимных гипертекстовых ссылок словаря терминов линейной алгебры (Linear Algebra Glossary, Google's Directory, www.google.com). Полное количество узлов-терминов равнялось 201. Для большей простоты мы сделали связи-ссылки ненаправленными. Вес каждой связи был равен 1. Компьютер выделил из полученного графа максимальную связную компоненту - неориентированный граф, который мы и рассматриваем ниже. Его основные данные: число узлов $n=197$, число связей $m=780$, средняя степень $2 m / n=7.9$, максимальная степень 54 , минимальная 1 , максимальное расстояние между двумя узлами (диаметр) 6, среднее расстояние по всем парам узлов 2.9 .

Вычисление $\bar{c}$ и $r=\lambda_{1}$ проводилось по формуле (3). При $\varepsilon=10^{-5}$ (см. раздел 2) потребовалось 16 итераций. Было найдено $r=14.3196\left(\lambda_{2}=8.43\right)$. Полученные 197 значений сердцевинности, в ранжировке по убыванию, приводятся в табл. 1.

Обращает на себя внимание дискриминирующая способность показателя сердцевинности: среди этих 197 значений $c$ нет одинаковых, тогда как степень узла имеет здесь лишь 26 различных значений ${ }^{1}$.

\footnotetext{
${ }^{1}$ Полные данные можно получить у авторов.
} 
ТАБЛИЦА 1

\begin{tabular}{|c|c|c|c|c|}
\hline № $\Pi / \Pi$ & $i$ & $c(i) / c_{\max }$ & $d(i)$ & Термин \\
\hline 1 & 46 & 1 & 54 & собственные значения \\
\hline 2 & 185 & 0.76 & 40 & симметричная матрица \\
\hline 3 & 84 & 0.64 & 31 & обратная матрица \\
\hline 4 & 47 & 0.56 & 26 & собственные векторы \\
\hline 5 & 146 & 0.55 & 28 & положительно определенная матрица \\
\hline 6 & 134 & 0.54 & 24 & ортогональная матрица \\
\hline 7 & 190 & 0.52 & 20 & транспонированная матрица \\
\hline 8 & 168 & 0.49 & 22 & вырожденная матрица \\
\hline ... & $\ldots$ & $\ldots$ & $\ldots$ & \\
\hline 197 & 3 & 0.003 & 1 & присоединенная (adjoint) матрица \\
\hline
\end{tabular}

$(d(i)$ - степень узла $i)$

Принимая в качестве сердцевины узлы, имеющие величину $c$ не ниже $50 \%$ от максимальной, получаем сердцевинный подграф, изображенный на рис. 1.

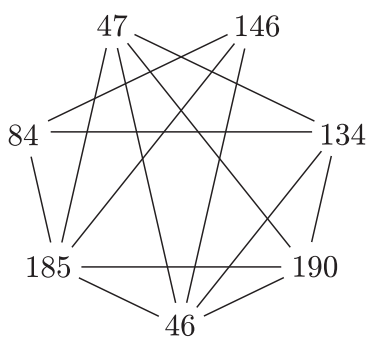

Рис. 1. Сердцевина графа, имеющего 197 узлов и 780 связей, - связный, плотный подграф на 7 узлах (14 связей из максимально возможного числа 21)

На рис. 2 и рис. 3 в логарифмических координатах показаны функции распределения сердцевинности и степени в данном графе (197 узлов). Кривые оказались похожими друг на друга. У каждой имеется линейно-образный "хвост", в более половины ее длины, т.е. участок, на котором $J(x) \sim x^{-a}$, где $a=$ const $>1$ (степенная функция распределения). В целом эти кривые довольно хорошо описываются функцией вида $J(x) \sim(b+x)^{-a}, b=$ const $>0$. Каждая кривая отражает наличие малой группы узлов, резко выделяющейся на фоне остальных. Например, в то время как более половины узлов (99/197) имеют сердцевинность лишь $11 \%$ или меньше от максимальной, сердцевинностью выше $50 \%$ от максимальной обладает группа, содержащая только $3.5 \%$ (7/197) узлов. Аналогично, степень выше $44 \%$ $(24 / 54)$ от максимальной имеют лишь $3.5 \%$ узлов, тогда как у $53 \%(105 / 197)$ из них она меньше $11 \%(6 / 54)$ от максимальной.

ПримеР 2. В предыдущем примере сердцевинный подграф обладал повышенной плотностью связей (близостью к полному графу), по сравнению с другими частями 


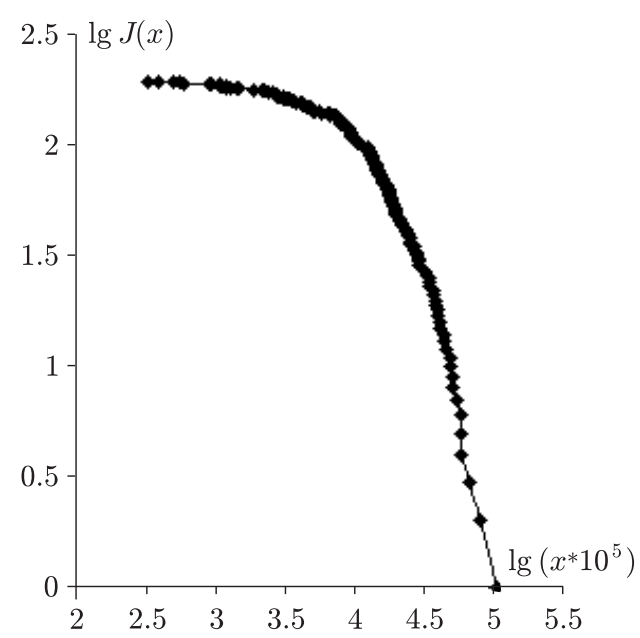

Рис. 2. $J(x)$ - число узлов, имеющих сердцевинность $c$ такую, что $c / c_{\max } \geqslant x$

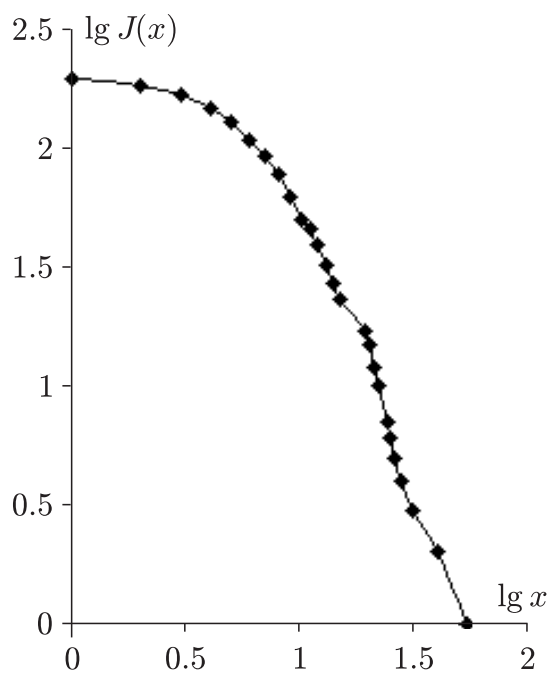

Рис. $3 . \quad J(x)$ - число узлов, имеющих степень $d \geqslant x$

сети. Подобное случается часто, но не всегда. Сеть может не иметь такой выделенной плотной зоны, обладая при этом вектором $\bar{c}$, ясно выделяющим узлы сердцевины. Последние тогда превосходят остальные узлы по маршрутной центральности (см. п. 3.1). Заметим также, что сердцевинный подграф "стремится" во всяком случае к связности (см. п. 3.2).

В качестве иллюстрации рассмотрим прямоугольную решетку $10 \times 7-$ неориентированный граф, имеющий $n=70$ узлов и $m=123$ связи. О какой-либо зоне, плотной по связям, здесь, очевидно, говорить не приходится. Что же касается показателя сердцевинности $c$, то он принимает здесь 20 различных значений; одинаковые значения $c$ имеют симметричные узлы. На рис. 4 узлы решетки помечены рангами от 1 до 20 в порядке убывания сердцевинности. Рядом приведен график функции распределения $c$.

\section{Заключение}

Величина показателя сердцевинности в узле сети характеризует положение узла в связочной структуре сети. Узлы с высокими значениями показателя - узлы сердцевины - соседствуют друг с другом и занимают центральное место в структуре. Соседство, “тесное расположение" узлов сердцевины превращает последнюю в плотное, насколько позволяет сеть, ядро - подграф, близкий к полному. Разреженная часть сети отмечена низкими значениями показателя, т.е. отнесена им к периферии. Центральность же узлов сердцевины означает, что они имеют высокий ранг по весу маршрутов, начинающихся в любом из этих узлов или кончающихся в нем.

Для движения с траекториями в виде маршрутов характерен дух исследования всех ассоциативных возможностей, а не стремление попасть оптимальным образом 

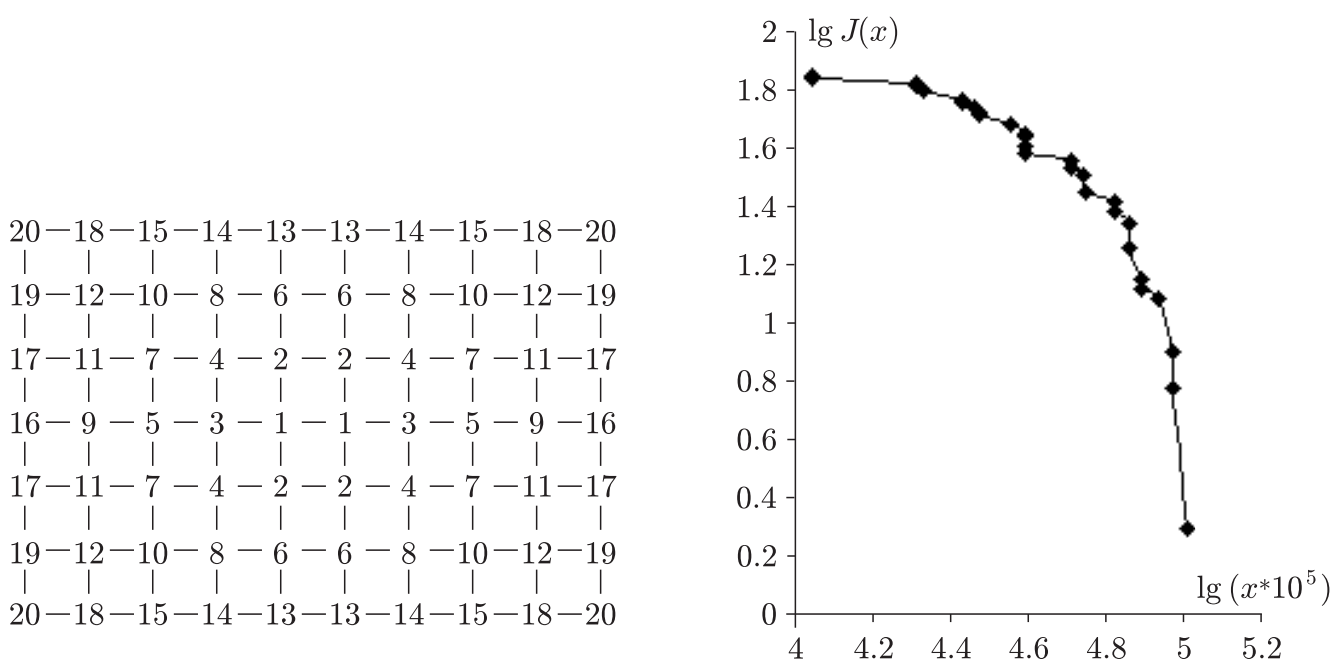

Рис. 4. Прямоугольная решетка с пометками узлов рангами по сердцевинности. $J(x)$ - число узлов сердцевинности $c$ такой, что $c / c_{\max } \geqslant x$

из пункта А в пункт Б. Наглядным примером здесь является перемещение пользователя по связям-ссылкам гипертекстовой сети, включая Всемирную Паутину, когда тема поиска существует лишь в общих чертах. Поисковая система Google использует рейтинг узлов Паутины (веб-страниц), даваемый финальными вероятностями случайного блуждания по сети. При стохастической сетевой матрице финальное распределение совпадает с вектором in-сердцевинностей узлов сети.

В сети, состоящей из информационных узлов и локальных смысловых связей, сердцевинность узла коррелирует с его принадлежностью к макроединицам смыслового содержания сети. Рейтинг узлов сети по "макровости" в сочетании с обходом узлов методом поиска в глубину позволяет получать связные тематические конспекты содержания сети [13], [14], что может быть использовано в автоматизированной системе реферирования.

Маршруты - это не только цепочки мысленных ассоциаций. К примеру, в социальных сетях по траекториям типа маршрутов распространяются новости, слухи, а также микробы заразных болезней [15]. Создаются мощные системы компьютерной имитации эпидемий в миллионных популяциях [16], [17]. Особое внимание в сетяхмоделях уделяется "хабам" - узлам с резко повышенным числом связей (hubs). Было бы интересно рассмотреть вместо них узлы сердцевины: при степенном распределении числа связей узла в сети сердцевина, скорее всего, совпадает с “хабами", но в других случаях последние могут и отсутствовать, в то время как сердцевина обычно хорошо различима (простой пример - сеть-решетка).

\section{СПИСОК ЦИТИРОВАННОЙ ЛИТЕРАТУРЫ}

[1] Р. Хорн, Ч. Джонсон, Матричный анализ, Мир, М., 1989.

[2] Дж. Голуб, Ч. Ван Лоун, Матричные вычисления, Мир, М., 1999. 
[3] Д. К. Фаддеев, В. Н. Фаддеева, Вычислительные методы линейной алгебры, Физматгиз, М., 1963.

[4] И. М. Гельфанд, Лекции по линейной алгебре, Наука, М., 1966.

[5] К. Берж, Теория графов и ее применения, ИЛ, М., 1962.

[6] Д. Цветкович, М. Дуб, Х. Захс, Спектры графов, Наукова думка, Киев, 1984.

[7] S. P. Borgatti, M. G. Everett, "Models of core/periphery structures", Social Networks, 21:4 (2000), 375-395.

[8] J. M. Kleinberg, "Authoritative sources in a hyperlinked environment", J. ACM, 46:5 (1999), 604-632.

[9] S. Brin, L. Page, "The anatomy of a large-scale hypertextual web-search engine", Proceedings of the 7th WWW Conference, Brisbane, Australia, 1998, 107-117.

[10] W. Kintsch, D. M. Welsch, "The construction-integration model: A framework for studying memory for text", Relating Theory and Data: Essays on Human Memory, eds. W. Hockley, S. Lewandowsky, Erlbaum, Hillsdale, NJ, 1991, 367-386.

[11] G. Storms, P. De Boeck, W. Ruts, "Prototype and exemplar-based information in natural language categories", J. Memory Language, 42:1 (2000), 51-73.

[12] В. В. Воеводин, Ю. А. Кузнецов, Матрицы и вычисления, Наука, М., 1984.

[13] V. M. Chelnokov, V. L. Zephyrova, "Hypertext macrodynamics", Lecture Notes in Computer Sci., 1015, Springer-Verlag, Berlin, 1995, 105-120.

[14] V. M. Chelnokov, V.L. Zephyrova, "Collective phenomena in hypertext networks", Proceedings of the Hypertext'97 Conference (Southhampton, UK), ACM, 1997, 220-221.

[15] S. P. Borgatti, "Centrality and AIDS", Connections, 18:1 (1995), 112-115.

[16] S. Eubank et al., "Modelling disease outbreakes in realistic urban social networks", Nature, 429:6988 (2004), 180-184.

[17] C.L. Barrett, S. G. Eubank, J.P. Smith, "If smallpox strikes Portland...", Scientific American, 292:3 (2005), 42-49.

В. М. Челноков

Межотраслевой научно-исследовательский институт "Интеграл" Федерального агентства по информационным технологиям Мининформсвязи РФ

\section{В. Л. Зефирова}

Межотраслевой научно-исследовательский институт

"Интеграл" Федерального агентства по информационным технологиям Мининформсвязи РФ

E-mail: victoria@smisc.net.ru
Поступило 14.09 .2005

Исправленный вариант 10.02.2006 